\title{
THERMO-VISCO-ELASTIC RAYLEIGH WAVES UNDER THE INFLUENCE OF COUPLE-STRESS AND GRAVITY
}

\author{
TAPAN KUMAR DAS and P. R. SENGUPTA
}

Indian Institute of Mechanics of Continua 201, Manicktala Main Road, Suite No. 42 Calcutta 700054, West Bengal, India

\section{and LOKENATH DEBNATH}

Department of Mathematics University of Central Florida Orlando, Florida 32816, U.S.A.

(Received August 10, 1989 and in revised form January 2, 1991)

\begin{abstract}
This paper is concerned with thermo-visco-elastic Rayleigh waves under the influence of couple-stresses and gravity. A more general phase velocity equation for these waves is derived. It is shown that the phase velocity equation reduces to that of the classical elastic Rayleigh waves in the absence of the couple-stress parameter, viscosity and gravity.
\end{abstract}

KEY WORDS AND PHRASES. Rayleigh waves, and thermo-visco-elastic Rayleigh waves. 1980 AMS SUBJECT CLASSIFICATION CODE. 73D20.

\section{INTRODUCTION.}

Chadwick [1] has studied the propagation of thermo-elastic Rayleigh waves in an elasticmedium with the assumption that heat is radiated from the free plane boundary surface of the solids, the maximum temperature difference across the surface being always small. On the other hand, Biot [2] has developed a theory of initial stresses of hydrostatic in nature that are produced by the force of gravity. Based upon this theory, he has investigated the propagation of Rayleigh waves. Subsequently, Sengupta et al [3-6] have studied thermo-elastic Rayleigh waves under the influence of gravity.

Based upon the linearized theory of couple-stresses due to Mindlin and Tiersten [7], several authors including Mindlin [8], Sengupta and Chel [9-10], Tiwari [11] have considered a large number of elastic wave problems under different configurations. In spite of these studies, hardly any attention has been given to the propagation of thermo-visco-elastic Rayleigh waves under the joint influence of couple-stresses and gravity. 
The main objective of this paper is to study thermo-visco elastic Rayleigh waves under the joint influence of couple-stresses and gravity. A more general phase velocity equation for these waves is obtained. In the absence of couple-stresses, viscosity and gravity, this equation is found to reduce to that of the classical elastic Rayleigh waves.

\section{FORMULATION OF THE PROBLEM WITH THE EFFECT OF COUPLE-STRESS.}

We consider the rectangular Cartesian coordinate system of Oxyz with the origin $O$ at any point on the free plane boundary of the half-space $z \geq 0$ and the $z$-axis directed normal to the interior of the isotropic visco-elastic medium under the influence of couple-stresses having homogeneous properties. It is assumed that the visco-elastic medium is free to exchange heat with the region $z \geq 0$. In the absence of a disturbance, the medium is at absolute temperature $T_{o}$.

In order to study the visco-elastic Rayleigh surface waves under the action of a thermal field propagated in the direction of the $\mathrm{x}$-axis, we introduce displacement potentials $\phi$ and $\psi$ related to the displacements $u, v, w$ by the equations

$$
u=\frac{\partial \phi}{\partial x}-\frac{\partial \psi}{\partial z}, v=0, w=\frac{\partial \phi}{\partial z}+\frac{\partial \psi}{\partial x}
$$

where $\phi$ and $\psi$ are functions of $x, z$ and time $t$, and satisfy the following relations:

$$
\nabla^{2} \phi=\frac{\partial u}{\partial z}+\frac{\partial w}{\partial z}, \quad \nabla^{2} \psi=\frac{\partial w}{\partial x}-\frac{\partial u}{\partial z}, \quad \nabla^{2} \equiv \frac{\partial^{2}}{\partial x^{2}}+\frac{\partial^{2}}{\partial z^{2}}
$$

We write the displacement equations of motion in the visco-elastic medium under the influence of temperature as well as the effect of couple-stresses in the form [1]:

$$
\begin{aligned}
& \rho \frac{\partial^{2} u}{\partial t^{2}}=[(\lambda\left.\left.+\mu_{o}\right)+\left(\lambda_{1}+\mu_{1}\right) \frac{\partial}{\partial t}\right] \frac{\partial \Delta}{\partial x}+\left(\mu_{o}+\mu_{1} \frac{\partial}{\partial t}\right) \nabla^{2} u \\
&+\left(\eta_{o}^{*}+\eta_{1}^{*} \frac{\partial}{\partial t}\right) \nabla^{2}\left(\frac{\partial \Delta}{\partial x}-\nabla^{2} u\right)-\frac{\left(\beta_{o}+\beta_{1} \frac{\partial}{\partial t}\right)}{\kappa_{T}} \frac{\partial \theta}{\partial x} \\
& \rho \frac{\partial^{2} w}{\partial t^{2}}=\left[\left(\lambda_{o}+\mu_{o}\right)+\left(\lambda_{1}+\mu_{1}\right) \frac{\partial}{\partial t} \frac{\partial \Delta}{\partial z}+\left(\mu_{o}+\mu_{1} \frac{\partial}{\partial t}\right) \nabla^{2} w\right. \\
&+\left(\eta_{o}^{*}+\eta_{1}^{*} \frac{\partial}{\partial t}\right) \nabla^{2}\left(\frac{\partial \Delta}{\partial z}-\nabla^{2} w\right)-\frac{\left(\beta_{o}+\beta_{1} \frac{\partial}{\partial t}\right)}{\kappa_{T}} \frac{\partial \theta}{\partial z}
\end{aligned}
$$

where $\Delta \equiv \nabla^{2}, \quad \lambda_{n}, \mu_{n}(n=0)$ are the elastic parameters, $\lambda_{n}, \mu_{n}(n=1)$, are the parameters representing the viscosity and $\mu_{n}^{*}(n=0,1)$ are the constants characterizing the existence of couplestresses, $\kappa_{T}$ is the isothermal compressibility, $\rho$ is the material density and $\theta=T-T_{o}$ is the temperature difference with $T_{o}$ as the initial temperature.

Due to rise of temperature of the material it is observed that the visco-elastic parameters written as $\mu_{o}+\mu_{1} \frac{\partial}{\partial t}, \lambda_{o}+\lambda_{1} \frac{\partial}{\partial t}$ and the thermal parameters $\beta_{o}+\beta_{1} \frac{\partial}{\partial t}$ are ultimately time dependent due to the fact that these parameters depend on temperature, and temperature is a function of time. This implies that the thermal parameters $\beta_{o}, \beta_{1}$ are needed to describe the state of affairs in thermo-visco-elastic solids. 
Using (2.1 abc), we obtain the following field equations from (2.3) - (2.4) satisfied by $\phi, \psi$ and $\theta$ :

where

$$
\begin{aligned}
& \frac{\partial^{2} \phi}{\partial t^{2}}=\left(V_{o T}^{2}+V_{1 T}^{2} \frac{\partial}{\partial t}\right) \nabla^{2} \phi-\frac{\left(U_{o S}^{2}+U_{1}^{2} \frac{\partial}{\partial t}\right)}{\kappa_{T}} \theta \\
& \frac{\partial^{2} \psi}{\partial t^{2}}=\left(V_{o S}^{2}+V_{1 S}^{2} \frac{\partial}{\partial t}\right) \nabla^{2} \psi-\left(V_{o R}^{2}+V_{1 R}^{2} \frac{\partial}{\partial t}\right) \nabla^{4} \psi
\end{aligned}
$$

$$
\begin{gathered}
V_{o T}^{2}=\frac{\lambda_{o}+2 \mu_{o}}{\rho}, V_{1 T}^{2}=\frac{\lambda_{1}+2 \mu_{1}}{\rho}, U_{o S}^{2}=\frac{\beta_{o}}{\rho}, U_{1 S}^{2}=\frac{\beta_{1}}{\rho} \\
V_{o S}^{2}=\frac{\mu_{o}}{\rho}, V_{1 S}^{2}=\frac{\mu_{1}}{\rho}, V_{o R}^{2}=\frac{\eta_{o}^{*}}{\rho}, V_{1 R}^{2}=\frac{\eta_{1}^{*}}{\rho}
\end{gathered}
$$

and $\beta_{n}=\left(3 \lambda_{n}+2 \mu_{n}\right) \alpha_{t}(n=0,1)$, in which $\alpha_{t}$ is the coefficient of linear expansion of the solid. Furthermore, to determine $\theta$, we need the Fourier's law of heat conduction in an isotropic viscoelastic medium as

$$
\kappa \nabla^{2} \theta=\rho C_{v} \frac{\partial \theta}{\partial t}+\frac{\rho\left(U_{o S}^{2}+U_{1 S}^{2} \frac{\partial}{\partial t}\right)}{\kappa_{T}} T_{o} \frac{\partial}{\partial t}\left(\nabla^{2} \phi\right)
$$

where $\kappa$ is the thermal conductivity and $C_{v}$ is the specific heat of the body at constant volume.

The boundary is assumed to be stress free and couple-stress free so that

$$
\sigma_{31}=\sigma_{33}=\mu_{32}=0 \text { on } z=0
$$

where

$$
\begin{aligned}
& \sigma_{31}=\rho\left(V_{o S}^{2}+V_{1 S}^{2} \frac{\partial}{\partial t}\right)\left(2 \frac{\partial^{2} \phi}{\partial x \partial z}+\frac{\partial^{2} \psi}{\partial x^{2}}-\frac{\partial^{2} \psi}{\partial z^{2}}\right)+\rho\left(V_{o R}^{2}+V_{1 R}^{2}\right) \nabla^{4} \psi \\
& \sigma_{33}=\rho\left(V_{o T}^{2}+V_{1 T}^{2} \frac{\partial}{\partial t}\right) \nabla^{2} \phi+2 \rho\left(V_{o S}^{2}+V_{o S}^{2} \frac{\partial}{\partial t}\right)\left(\frac{\partial^{2} \psi}{\partial x \partial z}-\frac{\partial^{2} \phi}{\partial x^{2}}\right)-\frac{\rho\left(U_{o S}^{2}+U_{1 S}^{2} \frac{\partial}{\partial t}\right)}{\kappa_{T}} \theta \\
& \mu_{32}=-2 \rho\left(V_{o R}^{2}+V_{1 R}^{2} \frac{\partial}{\partial t}\right) \nabla^{2}\left(\frac{\partial \psi}{\partial z}\right)
\end{aligned}
$$

Since the temperature difference across the free surface $z=0$ is always small, the linearized form of radiative condition is valid [1], and we have the thermal boundary condition

where $h$ is a constant.

$$
\frac{\partial \theta}{\partial z}+h \theta=0 \text { at } z=0
$$

\section{SOLUTION OF THE PROBLEM}

We seek plane wave solutions of equations (2.5) - (2.6) and (2.9) in the form

$$
(\phi, \psi, \theta)=(\Phi, \Psi, \Theta) \exp [i(k x-\omega t)]
$$

where $\Phi, \Psi$ and $\Theta$ are functions of $z$ only.

Substitution of $(3.1 \mathrm{abc})$ into $(2.5)-(2.6)$ and $(2.9)$ leads to the following differential equations

$$
\begin{gathered}
\frac{d^{2} \Phi}{d z^{2}}-\left[k^{2}-\frac{\omega^{2}}{\left(V_{o T}^{2}-i \omega V_{1 T}^{2}\right.}\right] \Phi=\frac{\left(U_{o S}^{2}-i \omega U_{1 S}^{2}\right)}{\kappa_{T}\left(V_{o T}^{2}-i \omega V_{1 T}^{2}\right)} \Theta \\
\frac{d^{2} \Theta}{d z^{2}}-\left(k^{2}-\frac{i \omega \rho C_{v}}{\kappa}\right) \Theta=-\frac{i \omega \rho T_{o}}{\kappa \kappa}\left(U_{o S}^{2}-i \omega U_{1 S}^{2}\right)\left(\frac{d^{2} \Phi}{d z^{2}}-k^{2} \Phi\right) \\
\frac{d^{4} \Psi}{d z^{4}}-\left(2 k^{2}+\frac{1}{\ell^{2}} \frac{d^{2} \Psi}{d z^{2}}+\left[k^{4}+\frac{k^{2}}{\ell^{2}}-\frac{\omega^{2}}{\ell^{2}\left(V_{o S}^{2}-i \omega V_{1 S}^{2}\right)}\right] \Psi=0\right.
\end{gathered}
$$


where $\ell$ is the couple-stress parameter and

$$
\ell^{2}=\frac{V_{o R}^{2}-i \omega V_{1 R}^{2}}{V_{o S}^{2}-i \omega V_{1 S}^{2}}
$$

Clearly, equations (3.2) - (3.4) must have surface wave solutions with exponentially decaying amplitude as $z \rightarrow \infty$. Hence the solutions for $\Phi, \Psi$ and $\Theta$ must have the form:

$$
\begin{gathered}
\Phi=\left[A e^{-z \sqrt{k^{2}-m_{1}^{2}}}+B e^{-z \sqrt{k^{2}-m_{2}^{2}}}\right] \exp [i(k x-\omega t)] \\
\Psi=\left[C e^{-z \sqrt{k^{2}-m_{3}^{2}}}+D e^{-z \sqrt{k^{2}-m_{4}^{2}}}\right] \exp [i(k x-\omega t)] \\
\Theta=\left[A_{1} e^{-z \sqrt{k^{2}-m_{1}^{2}}}+B_{1} e^{-z \sqrt{k^{2}-m_{2}^{2}}}\right] \exp [i(k x-\omega t)]
\end{gathered}
$$

where the square root with positive real part is taken and $m_{1}^{2}, m_{2}^{2}$ are the roots of the equation

with

$$
m^{4}-\left[\frac{\omega^{2}}{\left(V_{o T}^{2}-i \omega V_{1 T}^{2}\right)}+\frac{i \omega \rho C_{v}}{\kappa}(1+\epsilon)\right] m^{2}+\frac{\imath \omega^{3} \rho C_{v}}{\kappa\left(V_{o T}^{2}-i \omega V_{1 T}^{2}\right)}=0
$$

and $\dot{m}_{3}^{2}$ and $m_{4}^{2}$ are the roots of the equation

$$
\begin{aligned}
& \epsilon \equiv T_{o}\left(U_{o S}^{2}-i \omega U_{1 S}^{2}\right) / C_{v} \kappa_{T}^{2}\left(V_{o T}^{2}-i \omega V_{1 T}^{2}\right) \\
& \text { of the equation }
\end{aligned}
$$

$$
m^{4}-\frac{m^{2}}{\ell^{2}}-\frac{\omega^{2}}{\ell^{2}\left(V_{o S}^{2}-i \omega V_{1 S}^{2}\right)}=0
$$

The constants $A_{1}, B_{1}$ are related to the constants $A$ and $B$ through $A_{1}=\alpha_{1} A$ and $B_{1}=\alpha_{2} B$ where $\alpha, s$ are given by

$$
\alpha_{\jmath}=\frac{\kappa_{T}\left(V_{o T}^{2}-i \omega V_{1 T}^{2}\right)}{\left(U_{o S}^{2}-i \omega U_{1 S}^{2}\right)}\left[\frac{\omega^{2}}{\left(V_{o T}^{2}-i \omega V_{1 T}^{2}\right)}-m_{\jmath}^{2}\right], j=1,2
$$

Application of the boundary conditions (2.11) - (2.15) gives

$$
\begin{gathered}
{\left[2-\frac{\omega^{2}}{\left(V_{o S}^{2}-i \omega V_{1 S}^{2}\right) k^{2}}\right] A+\left[2-\frac{\omega^{2}}{\left(V_{o S}^{2}-i \omega V_{1 S}^{2}\right) k^{2}}\right]+\left(-2 i \beta_{3}\right) C+\left(-2 i \beta_{4}\right) D=0} \\
\left.\left(2 i \beta_{1}\right) A+\left(2 i \beta_{2}\right) B+\left[\left(1+\beta_{3}^{2}\right)-\ell^{2} k^{2}\left(1-\beta_{3}^{2}\right)^{2}\right] C+\left[1+\beta_{4}^{2}\right)-\ell^{2} k^{2}\left(1-\beta_{4}^{2}\right)^{2}\right] D=0 \\
\left(1-\beta_{3}^{2}\right) \beta_{3} C+\left(1-\beta_{4}^{2}\right) \beta_{4} D=0 \\
\left(\frac{h}{k}-\beta_{1}\right)\left[\beta_{1}^{2}-1+\frac{\omega^{2}}{\left(V_{o T}^{2}-i \omega V_{1 T}^{2}\right) k^{2}}\right] A+\left(\frac{h}{k}-\beta_{2}\right)\left[\beta_{2}^{2}-1+\frac{\omega^{2}}{\left(V_{o T}^{2}-i \omega V_{1 T}^{2}\right) k^{2}}\right] B=0
\end{gathered}
$$

where

$$
\beta_{j}^{2}=1-\left(m_{j}^{2} / k^{2}\right), \quad j=1,2,3,4
$$

The conditions of consistency between the homogeneous equations (3.13) - (3.16) lead to

where

$$
\begin{aligned}
& {\left[2-\frac{\omega^{2}}{\left(V_{o S}^{2}-i \omega V_{1 S}^{2}\right) k^{2}}\right]\left[\beta_{1}^{2}+\beta_{1} \beta_{2}+\beta_{2}^{2}-1+\frac{\omega^{2}}{\left(V_{o T}^{2}-i \omega V_{1 T}^{2} k^{2}\right.}\right] R-4 \beta_{1} \beta_{2} \beta_{3} \beta_{4}\left(\beta_{1}+\beta_{2}\right)\left(\beta_{3}+\beta_{4}\right)} \\
& \quad=\frac{h}{k}\left[\left\{2-\frac{\omega^{2}}{\left(V_{o S}^{2}-i \omega V_{1 S}^{2}\right) k^{2}}\right\}\left(\beta_{1}+\beta_{2}\right) R-4 \beta_{3} \beta_{4}\left(\beta_{3}+\beta_{4}\right)\left\{\beta_{1} \beta_{2}+1-\frac{\omega^{2}}{\left(V_{o T}^{2}-i \omega V_{1 T}^{2}\right) k^{2}}\right\}\right]
\end{aligned}
$$

$$
R=\left[\left(\beta_{3}+\beta_{4}\right)^{2}+\beta_{3}^{2} \beta_{4}^{2}-1+\ell^{2} k^{2}\left(1-\beta_{3}^{2}\right)\left(1-\beta_{4}^{2}\right)\left(1+\beta_{3} \beta_{4}\right)\right] .
$$


The above analysis reveals that equation (3.18) with (3.9) - (3.11) and (3.17) represents the dispersion relation of the thermo-visco-elastic Rayleigh surface waves. Obviously, $c=\omega / k$ is the velocity of the wave propagation which includes the effect of the couple-stresses. The dispersion equation (3.18) can be examined numerically for various values of the couple-stress parameter and the radiative condition of the temperature depending on the nature of the material and its constitutive relations. It may be noted that the wave velocity increases with the increase of the couple-stress parameter $\ell$. Equation (3.18) reduces to the corresponding result for the thermoelastic waves studied by Chadwick [1] when the couple-stress parameter and viscosity parameter are small. In the absence of the temperature field, the wave velocity equation is in excellent agreement with that of the corresponding classical elastic Rayleigh waves.

\section{SOLUTION OF THE PROBLEM WITH THE JOINT EFFECTS OF COUPLE-STRESS} AND GRAVITY.

In this section, we consider the joint effects of couple-stresses and gravity on the thermo-viscoelastic Rayleigh surface waves. We assume that gravitational field produces a type of initial stress of hydrostatic nature. Also, the initial stress is being produced by a slow process of creep where the shearing stresses tend to become small or vanish after a long period of time.

Based upon Biot's theory [3] of initial stresses, we use the following results for the present twodimensional problem

where $S$ is a function of depth.

$$
S_{11}=S_{33}=S, \quad S_{13}=0
$$

The equilibrium equation of the initial stress field can be obtained from Biot's theory in the form

$$
\frac{\partial S}{\partial x}=0, \quad \frac{\partial S}{\partial z}+\rho g=0
$$

where $\rho$ is the density and $g$ is the acceleration due to gravity.

The displacement equations of motion in the visco-elastic medium under the influence of temperature, couple-stress and gravity can be written (see $[1,4])$ as

$$
\begin{aligned}
\rho \frac{\partial^{2} u}{\partial t^{2}}= & {\left[\left(\lambda_{o}+\mu_{o}\right)+\left(\lambda_{1}+\mu_{1}\right) \frac{\partial \Delta}{\partial x}+\left(\mu_{o}+\mu_{1} \frac{\partial}{\partial t}\right) \nabla^{2} u\right.} \\
& +\left(\eta_{o}^{*}+\eta_{1}^{*} \frac{\partial}{\partial t}\right) \nabla^{2}\left(\frac{\partial \Delta}{\partial x}-\nabla^{2} u\right)-\frac{\left(\beta_{o}+\beta_{1} \frac{\partial}{\partial t}\right)}{\kappa_{T}} \frac{\partial \theta}{\partial x}+\rho g \frac{\partial w}{\partial x} \\
\rho \frac{\partial^{2} w}{\partial t^{2}}= & {\left[\left(\lambda_{o}+\mu_{o}\right)+\left(\lambda_{1}+\mu_{1}\right) \frac{\partial}{\partial t} \frac{\partial \Delta}{\partial z}+\left(\mu_{o}+\mu_{1} \frac{\partial}{\partial t}\right) \nabla^{2} w\right.} \\
& +\left(\eta_{o}^{*}+\eta_{1}^{*} \frac{\partial}{\partial t}\right) \nabla^{2}\left(\frac{\partial \Delta}{\partial z}-\nabla^{2} w\right)-\frac{\left.\beta_{o}+\beta_{1} \frac{\partial}{\partial t}\right)}{\kappa_{T}} \frac{\partial \theta}{\partial z}-\rho g \frac{\partial u}{\partial x}
\end{aligned}
$$

Using (1.1abc), we obtain from (4.3) - (4.4) the following sets of field equations satisfied by $\phi, \psi$ and $\theta$

$$
\begin{gathered}
\frac{\partial^{2} \phi}{\partial t^{2}}=\left(V_{o T}^{2}+V_{1}^{2} \frac{\partial}{\partial t}\right) \nabla^{2} \phi-\frac{\left(U_{o S}^{2}+U_{1 S}^{2} \frac{\partial}{\partial t}\right)}{\kappa_{T}} \theta+g \frac{\partial \psi}{\partial x} \\
\frac{\partial^{2} \psi}{\partial t^{2}}=\left(V_{o S}^{2}+V_{1 S}^{2} \frac{\partial}{\partial t}\right) \nabla^{2} \psi-\left(V_{o R}^{2}+V_{1 R}^{2} \frac{\partial}{\partial t}\right) \nabla^{4} \psi-g \frac{\partial \phi}{\partial x}
\end{gathered}
$$

where $V_{o T}^{2}, V_{1 T}^{2}, V_{o S}^{2}, V_{1 S}^{2}, V_{o R}^{2}, V_{1 R}^{2}, U_{o S}^{2}, U_{1 S}^{2}$ given by (2.7) - (2.8). We have also Fourier's law of heat conduction in an isotropic visco-elastic medium as presented in equation (2.9). 
Since the surface $z=0$ is free from both stresses and couple-stresses, the components of stresses and couple-stresses on the boundary $z=0$ are zero and the initial stresses due to gravity on $z=0$ are also zero, we have the following boundary conditions:

where

$$
S_{31}=S_{33}=\mu_{32}=0, \text { on } z=0
$$

$$
\begin{aligned}
& S_{31}=\rho\left(V_{o S}^{2}+V_{1 S}^{2} \frac{\partial}{\partial t}\right)\left(2 \frac{\partial^{2} \phi}{\partial x \partial z}+\frac{\partial^{2} \psi}{\partial x^{2}}-\frac{\partial^{2} \psi}{\partial z^{2}}\right)+\rho\left(V_{2 R}^{2}+V_{1 R}^{2} \frac{\partial}{\partial t}\right) \nabla^{4} \psi \\
& S_{33}=\rho\left(V_{o T}^{2}+V_{1 T}^{2} \frac{\partial}{\partial t}\right) \nabla^{2} \phi+2 \rho\left(V_{o S}^{2}+V_{1 S}^{2} \frac{\partial}{\partial t}\right)\left(\frac{\partial^{2} \psi}{\partial x \partial z}-\frac{\partial^{2} \phi}{\partial x^{2}}\right)-\rho \frac{\left(U_{o S}^{2}+U_{1 S}^{2} \frac{\partial}{\partial t}\right) \theta}{\kappa_{T}} \\
& \mu_{32}=-2 \rho\left(V_{o R}^{2}+V_{1 R}^{2} \frac{\partial}{\partial t}\right) \nabla^{2}\left(\frac{\partial \psi}{\partial z}\right)
\end{aligned}
$$

We also use the same thermal boundary condition as stated in equation (2.14). We seek solutions of equations (4.5)-(4.6) and (2.9) in the form

$$
[\phi, \psi, \theta]=\left[\Phi^{*}, \Psi^{*}, \Theta^{*}\right] \exp [i(k x-\omega t)]
$$

where $\Phi^{*}, \Psi^{*}$ and $\Theta^{*}$ are functions of $z$ only.

We substitute (4.11abc) into (4.5)-(4.6) and (2.9) to obtain the following differential equations

$$
\begin{gathered}
\frac{d^{2} \Phi^{*}}{d z^{2}}-\left[k^{2}-\frac{\omega^{2}}{\left(V_{o T}^{2}-i \omega V_{1 T}^{2}\right)}\right] \Phi^{*}=\frac{1}{V_{o T}^{2}-i \omega V_{1 T}^{2}}\left[\frac{\left(U_{o S}^{2}-i \omega U_{1 S}^{2}\right)}{\kappa_{T}} \Theta^{*}-i g k \Psi^{*}\right] \\
\frac{d^{4} \Psi^{*}}{d z^{4}}-\left(2 k^{2}+\frac{1}{\ell^{2}}\right) \frac{d^{2} \Psi^{*}}{d z^{2}}+\left[k^{4}+\frac{k^{2}}{\ell^{2}}-\frac{\omega^{2}}{\ell^{2}\left(V_{o S}^{2}-i \omega V_{1 S}^{2}\right)}\right] \Psi^{*}=-\frac{i g k \Phi^{*}}{\ell^{2}\left(V_{o S}^{2}-i \omega V_{1 S}^{2}\right)} \\
\frac{d^{2} \Theta^{*}}{d z^{2}}-\left(k^{2}-\frac{i \omega \rho C_{v}}{\kappa}\right) \Theta^{*}=-\frac{i \omega \rho T_{o}}{\kappa \kappa_{T}}\left(U_{o S}^{2}-i \omega U_{1 S}^{2}\right)\left(\frac{d^{2} \Phi^{*}}{d z^{2}}-k^{2} \Phi^{*}\right)
\end{gathered}
$$

where $\ell^{2}$ is given by (3.5).

We require $(4.11 \mathrm{abc})$ to represent surface waves with exponentially decaying amplitude as $z \rightarrow \infty$. This condition leads the solutions to assume the form

$$
\begin{aligned}
& \phi=\left[A^{\prime} e^{-z \sqrt{k^{2}-n_{1}^{2}}}+B^{\prime} e^{-z \sqrt{k^{2}-n_{2}^{2}}}+C^{\prime} e^{-z \sqrt{k^{2}-n_{3}^{2}}}+D^{\prime} e^{-z \sqrt{k^{2}-n_{4}^{2}}}\right] \exp [i(k x-\omega t)] \\
& \psi=\left[A_{1}^{\prime} e^{-z \sqrt{k^{2}-n_{1}^{2}}}+B_{1}^{\prime} e^{-z \sqrt{k^{2}-n_{2}^{2}}}+C_{1}^{\prime} e^{-z \sqrt{k^{2}-n_{3}^{2}}}+D_{1}^{\prime} e^{-z \sqrt{k^{2}-n_{4}^{2}}}\right] \exp [i(k x-\omega t)] \\
& \theta=\left[A_{2}^{\prime} e^{-z \sqrt{k^{2}-n_{1}^{2}}}+B_{2}^{\prime} e^{-z \sqrt{k^{2}-n_{2}^{2}}}+C_{2}^{\prime} e^{-z \sqrt{k^{2}-n_{3}^{2}}}+D_{2}^{\prime} e^{-z \sqrt{k^{2}-n_{4}^{2}}}\right] \exp [i(k x-\omega t)]
\end{aligned}
$$

Substituting the values of (4.15)-(4.17) into (4.12)-(4.14), it follows (4.12)-(4.14) that the constants in the solutions are related as follows:

$$
\begin{aligned}
& A_{1}^{\prime}=\alpha_{1}^{\prime} A^{\prime}, B_{1}^{\prime}=\alpha_{2}^{\prime} B^{\prime}, C_{1}^{\prime}=\alpha_{3}^{\prime} C^{\prime}, D_{1}^{\prime}=\alpha_{4}^{\prime} D^{\prime} \\
& A_{2}^{\prime}=\gamma_{1}^{\prime} A^{\prime}, B_{2}^{\prime}=\gamma_{2}^{\prime} B^{\prime}, C_{2}^{\prime}=\gamma_{3}^{\prime} C^{\prime}, D_{2}^{\prime}=\gamma_{4}^{\prime} D^{\prime} \\
& \alpha_{\jmath}^{\prime}=(i g k) /\left[\omega^{2}-n_{\jmath}^{2}\left(V_{o S}^{2}-i \omega V_{1 S}^{2}\right)-n_{j}^{4} \ell^{2}\left(V_{o S}^{2}-i \omega V_{1 S}^{2}\right)\right] \\
& \gamma_{\jmath}^{\prime}=\frac{i \omega \rho T_{o}\left(U_{o s}^{2}-i \omega U_{1 s}^{2}\right) n_{j}^{2}}{\left(i \omega \rho C_{v}-\kappa n_{\jmath}^{2}\right) \kappa_{T}}
\end{aligned}
$$

and $n_{j}^{2}(j=1,2,3,4)$ are the roots of the equation

$$
n^{8}+M_{1} n^{6}+M_{2} n^{4}+M_{3} n^{2}+M_{4}=0
$$


where

$$
\begin{gathered}
M_{1}=\left(\frac{1}{\ell^{2}}-N\right), \quad M_{2}=\left[p q-\frac{\omega^{2}}{\ell^{2}\left(V_{o S}^{2}-i \omega V_{1 S}^{2}\right)}-\frac{N}{\ell^{2}}\right], \quad N=\left(p+q-r q_{1}\right), \\
M_{3}=\left[\frac{\omega^{2} N}{\ell^{2}\left(V_{o S}^{2}-i \omega V_{1 S}^{2}\right)}+\frac{p q}{\ell^{2}}-s p_{1}, \quad M_{4}=\left[s p_{1} q-p q \frac{\omega^{2}}{\ell^{2}\left(V_{o S}^{2}-i \omega V_{1 S}^{2}\right)}\right],\right. \\
p=\frac{\omega^{2}}{\left(V_{o T}^{2}-i \omega V_{1 T}^{2}\right)}, \quad q=\frac{i \omega \rho C_{v}}{\kappa}, \quad r=\frac{\left(U_{o S}^{2}-i \omega U_{1 S}^{2}\right)}{\kappa_{T}\left(V_{o T}^{2}-i \omega V_{1 T}^{2}\right)}, \\
s=\frac{i g k}{\left(V_{o T}^{2}-\omega V_{1 T}^{2}\right)}, \quad p_{1}=\frac{i g k}{\ell^{2}\left(V_{o S}^{2}-i \omega V_{1 S}^{2}\right)}, \quad q_{1}=-\frac{i \rho \omega\left(U_{o S}^{2}-i \omega U_{1 S}^{2}\right) T_{o}}{\kappa \kappa T}
\end{gathered}
$$

The boundary conditions (4.7) and (2.15) yield the following results:

$$
\begin{aligned}
& \sum_{j=1}^{4} H j\left[2 i \beta^{\prime} j+\alpha_{j}^{\prime}\left(1+\beta_{j}^{\prime 2}\right)-\ell^{2} \alpha_{j}^{\prime} n_{j}^{2}\left(1-{\beta_{j}^{\prime}}^{2}\right)\right]=0 \\
& \sum_{\jmath=1}^{4} H j\left[\rho\left(V_{o T}^{2}-i \omega V_{1 T}^{2}\left(1-\beta_{\jmath}^{\prime 2}\right)+2 \rho\left(V_{o S}^{2}-i \omega V_{1 S}^{2}\right)\left(i \alpha_{\jmath}^{\prime} \beta_{\jmath}^{\prime}-1\right)+\frac{\rho\left(U_{o S}^{2}-i \omega U_{1 S}^{2}\right) \gamma_{\jmath}^{\prime}}{k^{2} \kappa_{T}}\right]=0\right. \\
& \sum_{j=1}^{4} H_{j}\left[\alpha_{j}^{\prime} \beta_{j}^{\prime}\left(1-\beta_{j}^{\prime 2}\right)\right]=0 \\
& \sum_{j=1}^{4} H_{j}\left[\gamma_{j}^{\prime}\left(\frac{h}{k}-\beta_{j}^{\prime}\right)\right]=0
\end{aligned}
$$

where

$$
\beta_{j}^{2}=1-\frac{n_{j}^{2}}{k^{2}} ; \quad j=1,2,3,4
$$

and

$$
A^{\prime}=H_{1}, \quad B^{\prime}=H_{2}, \quad C^{\prime}=H_{3}, \quad D^{\prime}=H_{4} .
$$

The condition of consistency between the homogeneous equations $(4.22)-(4.25)$ is given by the following determinant

$$
\left|a_{\kappa j}\right|=0 \quad(\kappa, j=1,2,3,4)
$$

where

$$
\begin{gathered}
a_{1 \jmath}=\left[2 i \beta_{\jmath}^{\prime}+\alpha_{\jmath}^{\prime}\left(1+{\beta_{\jmath}^{\prime}}^{2}\right)-\ell^{2} \alpha_{\jmath}^{\prime} n_{\jmath}^{2}\left(1-\beta_{j}^{\prime 2}\right)\right] \\
a_{2 \jmath}=\left[\rho\left(V_{o T}^{2}-i \omega V_{1 T}^{2}\right)\left(1-\beta_{\jmath}^{\prime}\right)+2 \rho\left(V_{o S}^{2}-i \omega V_{1 S}^{2}\right)\left(i \alpha_{\jmath}^{\prime} \beta_{\jmath}^{\prime}-1\right)+\frac{\rho\left(U_{o S}^{2}-i \omega U_{1 S}^{2}\right) \gamma_{j}^{\prime}}{k^{2} \kappa_{T}}\right] \\
a_{3 \jmath}=\left[\alpha_{\jmath}^{\prime} \beta_{j}^{\prime}\left(1-{\beta_{\jmath}^{\prime}}_{\jmath}^{2}\right)\right], a_{4 \jmath}=\left[\gamma_{j}^{\prime}\left(\frac{h}{k}-\beta_{\jmath}^{\prime}\right)\right]
\end{gathered}
$$

It is noted that equation (4.27) represents the phase velocity equation for the thermo viscoelastic Rayleigh waves under the influence of couple-stresses and gravity. This equation can be studied numerically for various values of couple-stress parameter and the radiative conditions characterizing the temperature effect. In the absence of gravitational effect, the phase velocity equation (4.27) is in perfect agreement with the phase velocity equation of the last section. When viscosity, gravity, and couple-stress effects are neglected, the phase velocity equation (4.27) reduces to the thermo-elastic Rayleigh waves as presented by Chadwick [1].

ACKNOWLEDGEMENT: This work is partially supported by the University of Central Florida. 


\section{REFERENCES}

1. CHADWICK, P., Progress in Solid Mechanics, Vol. I (Ed : I. N. Sneddon and R. Hill) NorthHolland Publ. Co., Amsterdam (1960) p. 263.

2. BIOT, M.A., Mechanics of Incremental Deformations, New York (1965).

3. SENGUPTA, P.R. and GHOSH, B.C., Ger. Beitr. Geophys., Leipzig 83 ( 1974) 4, 309-318.

4. SENGUPTA, P.R. and ACHARYA, D.P., Acta Ciencia Indica, 2. No. 4 (1976) p. 406.

5. DE, S.N. and SENGUPTA, P.R., Ger. Beitr. Geophys., Leipzig 85 (1976) pp. 311-318.

6. DE, S.N. and SENGUPTA, P.R., Ger. Beitr. Geophys., Leipzig 84, 6 (1975) pp. 509-514.

7. MINDLIN, R.D. and TIERSTEN, H.F., Arch. Rat. Mech. Anal., 11 (1962) pp. 415-448.

8. MINDLIN, R.D., Exp. Mech. 3 (1963) pp. 1-7.

9. SENGUPTA, P.R. and CHEL, J.D., Ger. Beitr. Geophys., Leipzig 93 (1984) pp. 223-230.

10. SENGUPTA, P.R. and CHEL, J.D., Bull. Acad. Pol. Sci. Ser. Sci. Techn. 34, No. 11-12 (1986).

11. TIWARI, G.R., Ind. J. Eng. Math., (1968) pp. 63-67. 


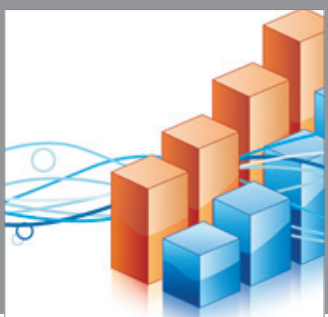

Advances in

Operations Research

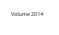

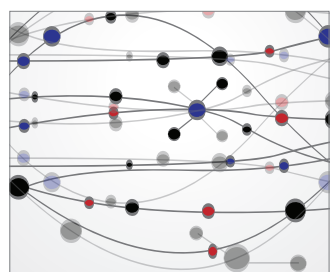

\section{The Scientific} World Journal
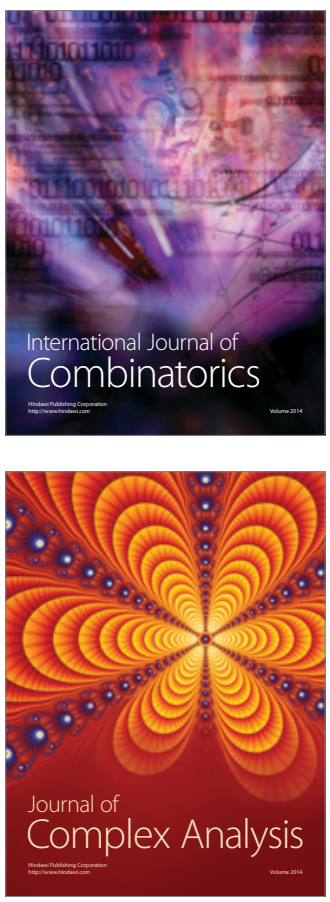

International Journal of

Mathematics and

Mathematical

Sciences
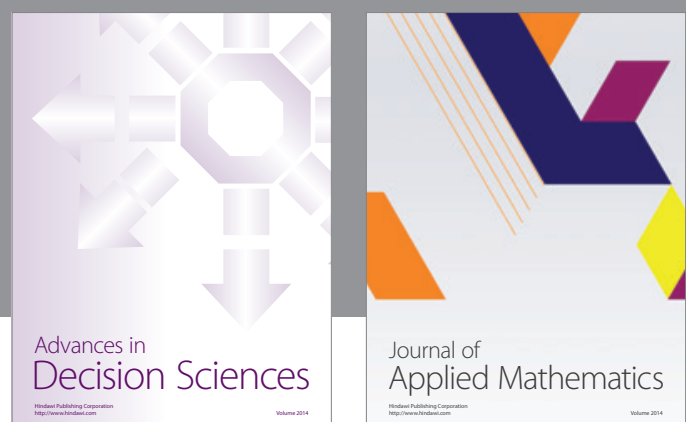

Journal of

Applied Mathematics
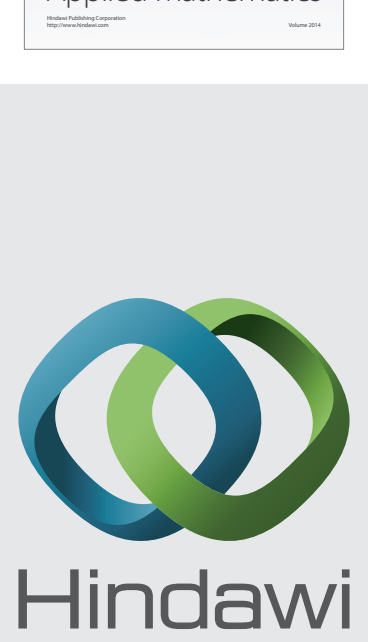

Submit your manuscripts at http://www.hindawi.com
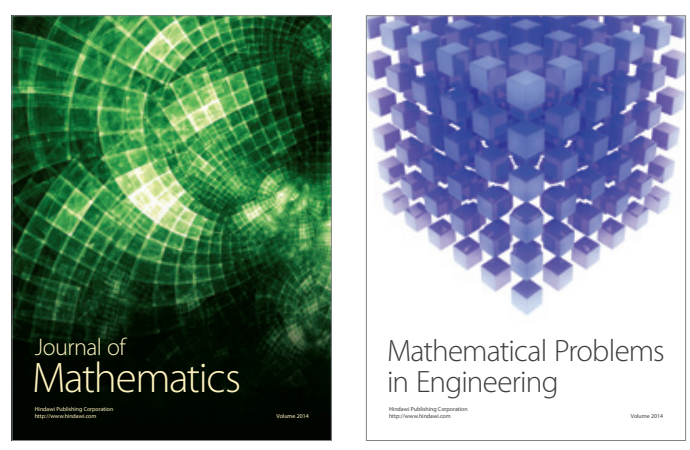

Mathematical Problems in Engineering
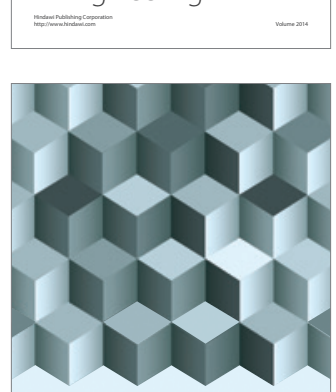

Journal of

Function Spaces
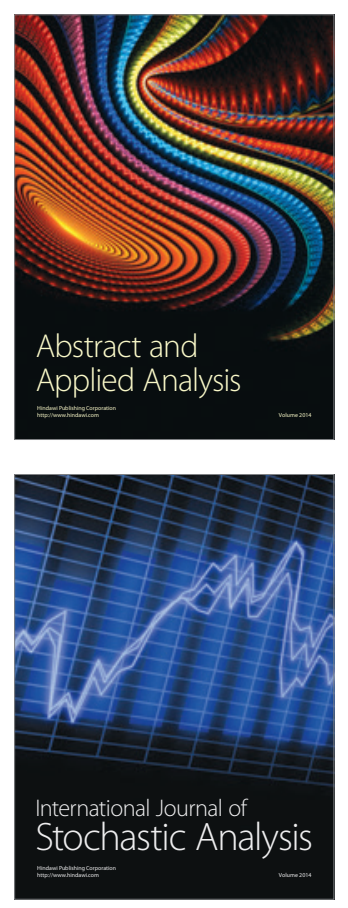

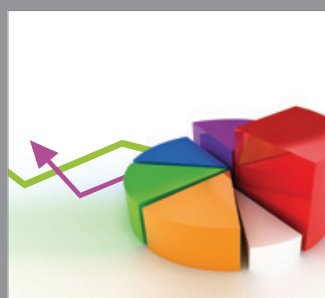

ournal of

Probability and Statistics

Promensencen
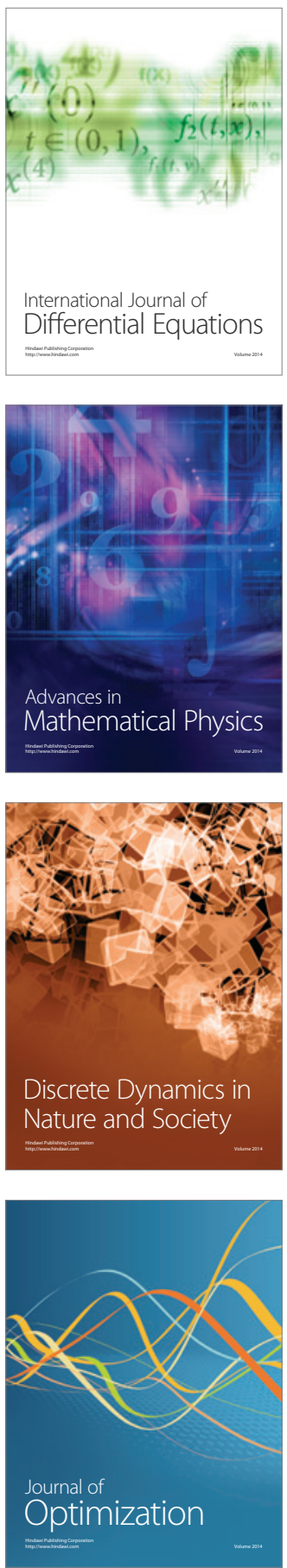